\title{
Alimentación enteral precoz en lactantes con infección respiratoria baja
}

\author{
Francisco Lagrutta $S .{ }^{1,2}$; Carlos Castillo D. ${ }^{1,3}$
}

\section{Early enteral feeding of infants with lower respiratory infections}

\begin{abstract}
A decrease in food intake during the acute phase of lower respiratory tract infections may affect the course of illness. To determine if enteral feeding improves the clinical course of lower respiratory infections (LRV) we studied 42 infants $(2$ to 12 months of agel with LRI which were given antibiotics and salbutamol theraphy based on clinical and $X$-ray findings. On admission to the hospital patients were randomly allocated to group $A$ ifed by nasogastric tube as intermittent bolus): group $B$ (infants fed by continous drip via nasogastric tube) and group $C$ (subjects fed ad-libitum by the oral routel. Feedings consisted of powdered whole cow's milk formula, reconstituted to $7.5 \%$ (W/V), with added sucrose $5 \%$ and maltadextrin $5 \%(77 \mathrm{kca} / \mathrm{d}$ d!). Energy intake in group A increased from 69 to $110 \mathrm{kcal} \cdot \mathrm{kg} \cdot$ day; in group B, from 68 to $114 \mathrm{kcal} \cdot \mathrm{kg} \cdot$ day, and in group C, from 55 to $109 \mathrm{kcal} \cdot \mathrm{kg} \cdot \mathrm{day}$ (ANOVA, $P<0.005$ for the first three days). Intants of groups $A$ and $B$ had shorter hospital stays than $C$ ( 8.7 and 8.4 vs. 9.8 davs, ANOVA, $p<0.02$ ) and a faster improvement of respiratory failure index $\{4.1$ and 3.8 vs. 5.5. days, $p<0.001$ ). Groups $A$ and $B$ also showed greater decrease in their heart rates (mean 170 down to 134 beats/minl than group $C$ patients 1169 down to 142 beats $/$ min), $p<0.01$ at discharge from the hospital, No differences were noted in trends of respiratory rate, weight/lenght, albuminemia, C-reactive protein, eritrosedimentation rate or glycernia. No adverse effects of nasogastric tube feeding were observed. Early nutrittonal support via a nasogastric tube seems to enhance clinical recovery from lower respiratory tract infections.

t Key words: enteral feding, low respiratory infections, infants.)
\end{abstract}

Las enfermedades respiratorias constituyen un importante problema de Salud Publica por su implicancia en la morbimortalidad pediátrica. En Chile, las enfermedades respiratorias son la principal causa de consulta de lactantes en centros de atención primarja y de urgencia y también de hospitalización', debida ésta a bronconeumonias $y$ afecciones bronquiales obstructivas. En el resto de Iberoamérica, la situación es similar ${ }^{2}$ La insuficiencia respiratoria, concomitante con las infecciones broncopulmonares más severas, en especial en los lactantes más pequeños, puede hacer necesario recurrir a ven. tilación asistida en unidades de cuidados intensivos y desembocar eventualmente en la muerte.

Desde el punto de vista nutricional la infección y la dificultad respiratoria reducen habitualmente la ingestión de alimentos, lo cual, a su vez, podría incidir sobre el curso de la enfermedad de base. Esta interacción podría ser aún

1. Instituto de Nutrición y Tecnotogía de los Alimentos, Universidad de Chile.

2. Hospital del Niño, Panamá, Panamá.

3. Servicio de Pediatría, Hospital Dr. Sótero del Río. de mayor importancia, cuando el paciente se enfrenta a la infección respiratoria en un estado nutricional previamente deteriorado.

El objetivo de este estudio fue establecer si el apoyo nutricional, mediante alimentación ente. ral desde el comienzo de la hospitalización, en lactantes con infección respiratoria baja e insuficiencia respiratoria, afecta el curso clínico de la enfermedad respiratoria y si produce efectos adversos.

\section{Sujetos y Métodos}

Se estudiaron 42 lactantex de ambos sexos ${ }_{1}$ de 2 a 12 meses de edad, seleccionados entre los pacientes que ingresaron al servicio de pediatrja del Hospital Sótero del Río de Santiago, Chile, en los meses de mayo a septiembre de 1989. Todos tenían infección respiratoria baja, básicamente bronconeumonía, diagnosticada clínica y radiográficamente -acompañada o no de síndrome bronquial obstructivo-, y signos de insuficiencia respiratoria severa, corroborada con presión parcial arterial de oxigeno $\left(\mathrm{paO}_{2}\right)$ menor de $60 \mathrm{~mm}$ de $\mathrm{Hg}$. Se excluyó a los lactantes que tuviesen signos de compromiso digestivo, enfermedades agregadas, estuvieran recibiendo lactancia materna o hubie- 
ran recibido antibióticos en las dos semanas previas a la hospitalización. Los nitios admitidos al estudio fueron alimentados con una fómula en base a leche de vaca entera en polvo, reconstituida a 7,5\% (peso/volumen) con sacarosa $5 \%$ y maltosa-dextrina $5 \%$, que aportaba $77 \mathrm{kcal} / \mathrm{dl}$. En forma aleatoria los pacientes fueron asignados al ingreso a uno de tres grupos. Los del grupo $A(n=15)$ fueron alimentados con bolos de la fórmula administrados cada $3 \mathrm{~h}$ por sonda nasogástrica permanente; a los del grupo $B(n=14)$ se les dio alimentación cada 3 horas por sonda nasogástrica, en goteo continuo a lo largo de 2 horas y media dejando 30 min sin alimentación antes del suministro siguiente; los nifrios del grupo $\mathrm{C}(\mathrm{n}=13)$ recibieron la fórmula por vía oral ad libitum cada 3 horas. Para los dos grupos que recibieron alimentación por sonda nasogástrica se planificó al primer día un aporte de $70 \mathrm{kcal} \cdot \mathrm{kg} \cdot$ día; al segundo $85 \mathrm{kcal} \cdot \mathrm{kg} \cdot$ día y al tercero $100 \mathrm{kcal} \cdot \mathrm{kg} \cdot \mathrm{d} \mathrm{a}_{\text {, }}$ intentando la alimentación por via ora ad libitum a partir del cuarto día de estudio. Todos fueron tratados con oxígeno por los primeros 2 ó 3 dias, broncodilatadores (salbutamol en nebulizaciones cada 4 horas), antibióticos (penicilina $G$ sódica), kinesiterapia respiratoria. Un niño de cada grupo recibió, además, corticoesteroides. Los pacientes fueron seguidos prospectivamente durante 7 días, incluso los fines de semana y festivos, por el mismo observador (FL). Se registró la frecuencia cardíaca con fonendoscopio durante tres intervalos de un minuto, entre las $8: 30 \mathrm{y}$ $9: 00$, al menos dos horas después de la última alimentación, estando el lactante despierto, sin llanto y retirando el aporte de oxígeno por un mínimo de 30 minutos; la frecuencia respiratoria fue obtenida en similares condiciones, así como la presencia de cianosis y esfuerzo respiratorio. Se midió el peso diario, la talla al ingreso y se extrajeion $4 \mathrm{ml}$ de sangre al ingreso y en el día 7 para análisis de proteína C-reactiva, albuminemia, velocidad de eritrosedimentación y glicemia. E1 manejo clínico terapéutico y la decisión de egreso hospitalario estuvo a cargo del equipo profesional tel hospital en base a las pautas habituales del servicjo de pediatría y en forma independiente y ciega de Jos investigadores. El análisis estadístico incluyó cálculos de promedio, desviación estándar, análisis de varianza y t de Student. Los resultados fueron analizados computacionalmente con el paquete estadístico SAS (Statistical Analysis System) en un aparato IBM.

\section{Resultados}

El estado nutricional de los 3 grupos al momento del ingreso era normal (relación $\mathrm{P} / \mathrm{T}$ de $97,0,96,9$ y $93,3 \%$; grupos A, B y C, respecti. vamente); la proporción de los sexos era semejante (M:F 6:9, 9:5 y 7:6, respectivamente) y la mediana de edad de 5 meses (límites: 2 a 10 ). La frecuencia de sindrome bronqujal obstructivo también fue parecida en los 3 grupos (7/15, $6 / 14,6 / 13)$.

No hubo diferencias significativas en la adecuación $\mathrm{P} / \mathrm{T}$ entre el ingreso y el egreso en los grupos A $(100,2$ vs $99,2 \%)$ y B (101,5 vs $101,7 \%)$. En el grupo $\mathrm{C}$ hubo un descenso algo mayor $(96,6$ a $94,9 \%$, t par. $p<0,0104)$. No se en. contró diferencias significativas intergrupales. Se resalta que los valores al ingreso, para los 3 grupos, eran normales. La ingestión de calorías (tabla 1) fue significativamente diferente en los tres primeros días, en concordancia con el protocolo de estudio, siendo mayor en los grupos A y B que en el C, pero a partir del día 4 ya no se observaron diferencias.

Tabla 1

Ingesta calórica diaria en tres grupos de lactantes con infecciones respiratorias bajas, durante los primeros seis días de hospitalización

\begin{tabular}{cccccccc}
\hline Día & 1 & 2 & 3 & 4 & 5 & \\
\hline A (kal $\cdot \mathrm{kg})$ & $68,8 \pm 2,8$ & $79,9 \pm 6,0$ & $88,5 \pm 9,3$ & $98,1 \pm 9,9$ & $104,8 \pm 7,6$ & $110,1 \pm 8,1$ \\
B (kal $\cdot \mathrm{kg})$ & $67,9 \pm 5,8$ & $79,6 \pm 7,7$ & $90,7 \pm 9,8$ & $97,5 \pm 9,4$ & $107,2 \pm 8,4$ & $113,6 \pm 11$ \\
C (kal $\cdot \mathrm{kg})$ & $54,6 \pm 5,2$ & $65,0 \pm 5,1$ & $80,2 \pm 4,7$ & $92,3 \pm 7,5$ & $102,2 \pm 9,1$ & $109,4 \pm 8,7$
\end{tabular}

Análisis de varianza $(\mathrm{p}<)$.

$\begin{array}{lll}(0,0001) & (0,0001) & (0,0054) \\ x A-x C & x A-x C & x A-x C \\ x B-x C & x B-x C & x B-x C\end{array}$

A: fórmula láctea en bolos por sonda nasogástrica.

B: fórmula láctea en gotèo contimuo por sonda nasogástrica,

C: fórmula láctea ad libitum por biberón. 


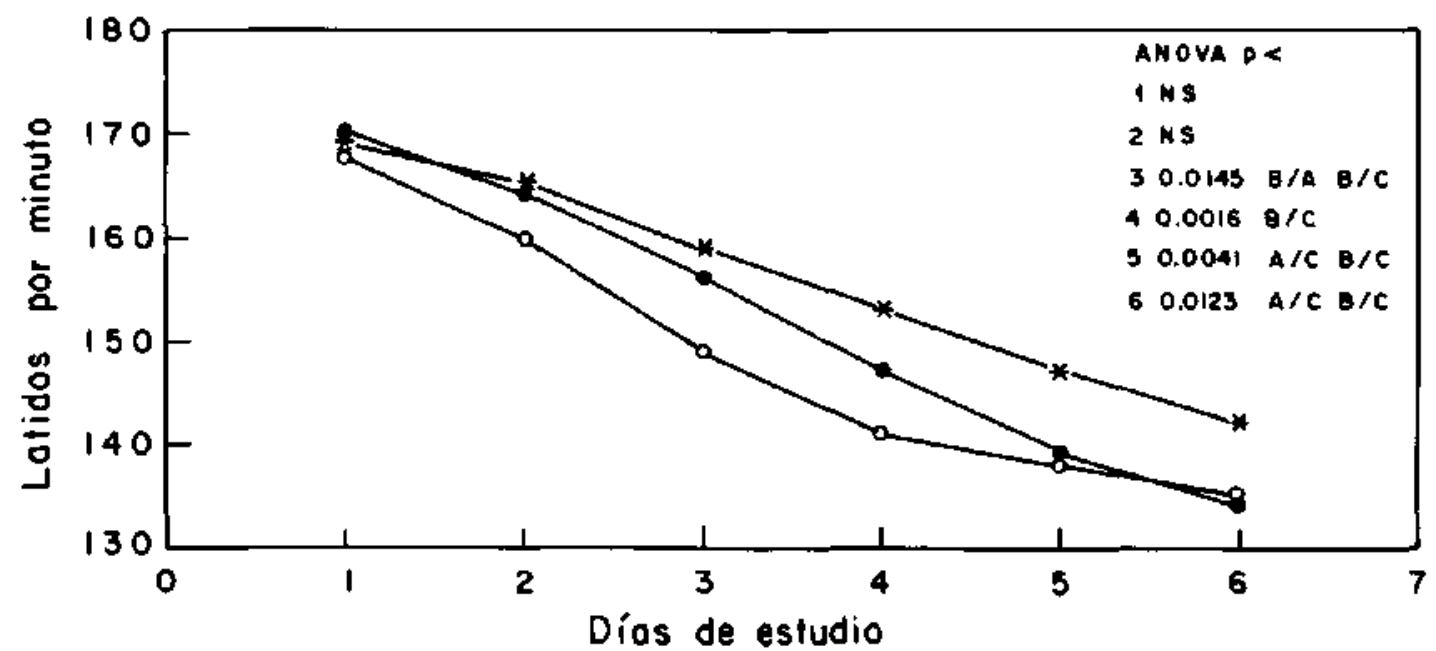

Figura 1: Frecuencia cardíaca matinal en hetantes hospitalizados con bronconeumonía, a través de los 6 primeros días de estadía, y según gupos alimentados con fórmula isocalórica: A, sonda nasogástrica y alimentación en bolo cada 3 horas (-; $\mathrm{n}=15$ ); $\mathrm{B}$, sonda nasogástrica y alimentación a goteo continua cada 3 horas (o-O; $n=14) ; \quad C$, alimentación cada 3 horas, oral ad libitum $(*-* ; n=13)$.

El promedio de la frecuencia cardíaca por día y por grupo (figura 1) fue semejante en los días I y 2 , pero desde el día 3 hasta el 6, los grupos A y B mostraron descenso significativamente mayor que el grupo $C$. No se encontró diferencias en los promedios de frecuencia respiratoria por día y por grupo.

Todos los grupos, con excepción del A, para la albuminemia (tabla 2), evidenciaron mejoria significativa en los valores de egreso de las variables bioquímicas, si bien todas ellas estaban desde el comienzo dentro de márgenes de normalidad. La glicemia (tabla 2), la velocidad de eritrosedimentación y la proteína C.reactiva (tabla 3) mostraron descensos significativos en todos los grupos, sin diferencias intergnupales.

El promedio de días de hospitalización fue menor en los grupos que recibieron leche por sonda nasogástrica $(8,7 \pm 1,5$ y $8,4 \pm 1,2$ días, grupos A y B vs $9,8 \pm 1,0$ días, grupo C). El análisis de varianza demostró que el promedio de los grupos A y B fue significativamente me.

Tabla 2

Concentraciones plasmáticas de albúmina y glucosa en tres grupos de lactantes con infecciones respiratorias bajas, al ingresar y egresar del hospital

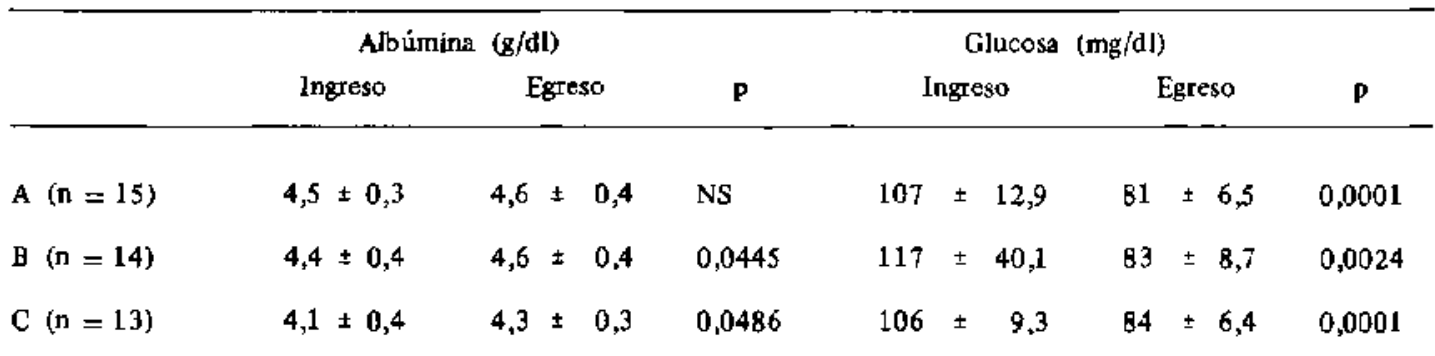

Análisis de varianzo NS.

A: fótmula láctea en bolos por sonda nasogástrica.

B: fórmula láctea en goteo continuo por sonda nasogástrica.

C: fór mula láctea ad libirum por biberón.

p: prueba $T$ de Student. 
Tahla 3

Velocidad de eritrosedimentación y proteína C-reactiva, en tres grupos de lactantes con infecciones respiratorias bajas al ingresar $y$ egresar del hospital

\begin{tabular}{|c|c|c|c|c|c|c|c|c|c|c|}
\hline & \multicolumn{3}{|c|}{ VHS $(\mathrm{mm} / \mathrm{h})$} & \multirow[b]{2}{*}{$\mathrm{p}$} & \multicolumn{5}{|c|}{$\mathrm{PCR}(\mathrm{mg} / \mathrm{l})$} & \multirow[b]{2}{*}{$\mathbf{p}$} \\
\hline & Ingreso & & reso & & & gres & & & greso & \\
\hline$A(n=15)$ & $36 \pm 35$ & 9 & $\pm 7,5$ & 0,0042 & 42 & \pm & 61 & 6 & \pm 10 & 0,0419 \\
\hline B $(n=14)$ & $35 \pm 23$ & 11 & $=9,8$ & 0,0001 & 42 & $=$ & 56 & 5 & $\pm 5,6$ & 0,0284 \\
\hline$C(n=13)$ & $40 \pm 25$ & 14 & \pm 10 & 0,0002 & 46 & \pm & 28 & 3 & $\pm 3,4$ & 0,0003 \\
\hline
\end{tabular}

Aválisis de varianza NS.

A: fórmula láctea en bolos por sonda nasogástrica.

B: fórmula láctea en goteo continuo por sonda nasogástrica.

C: fórmula láctea ad libitum por biberón.

p: prueba $T$ de Student.

VHS: velocidad de eritrosedimentación.

PCR: proteina C-reactiva.

nor $(\mathrm{p}<0,0216)$ al del grupo $\mathrm{C}$. No se encontró diferencias entre los grupos A y B. La cantidad promedio de días cursados con dificultad respi. ratoria severa fue, en los lactantes de los grupos A y B, significativamente menor que en el grupo C $(4,1 \pm 0,8$ días y $3,8 \pm 0,7$ días, grupos A y B vs $5,5 \pm 0,5$ dias, grupo $C, p<0,0001)$. No se encontró diferencias entre los gnupos A y B.

\section{Discusión}

Hemos estudiado la interrelación entre infec. ciones respiratorias agudas bajas y nutrición, motivados por la alta incidencia de ellas en njños -en especial las más graves, que requieren hospitalización y pueden causar muertes en lactantes-y por la posibilidad de modificar su evolución con herramientas nutricionales.

En estudios sobre la evolución natural de determinadas enfermedades y su incidencia sobre el estado de nutrición sin intervención nutricional, en lactantes que recibían fórmulas ad libitum por via oral, se ha encontrado evidente deterioro de los parámetros antropométricos y significativa asociación de hospitalización prolongada con valores menores de ellos, salvo, en ambos casos, talla/edad ${ }^{3,4}$. Estos hallazgos estimu. laban a estudiar el impacto de un apoyo nutricional efectivo sobre el estado de la nutrición durante las infecciones respiratorias severas. Nuestros resultados comprueban que en el transcurso de estas infecciones hay una capacjdad metabólica adecuada para utilizar lo nu. trientes que estén, al menos, cubriendo los re. querimientos normales; aportes mayores que los aceptados por un lactante alimentado ad libitum por via oral, le permiten mantener su estado nutricional. Al interpretar el indicador peso en esta situación durante el período de estado de la enfermedad, debe tenerse en cuenta la retención de agua propia del periodo agudo de las infecciones ${ }^{5}$, que puede estar ayudando a ocultar pérdidas de peso de magnitudes un poco mayores. Los indicadores antropométricos al ingreso, en este estudio como en otros, no fueron predictores de la duración de la hospitalización ${ }^{3}$, lo que podría atribuirse al buen estado nutricional de los sujetos al ingreso.

El parámetro que empleamos para evaluar el comportamiento de las proteínas viscerales, la albúmina del plasma, aumentó significativamente al egreso con respecto al ingreso (excepción para el grupo A). Se acepta que los niveles disminuidos de albúmina pueden reflejar depleción de las proteínas viscerales, baja disponibilidad de aminoácidos en el plasma, las dos anteriores ${ }^{4}$ o reuti. lización de proteínas para síntesis de las llamadas "proteinas de fase aguda" y otros factores de inmunidad ${ }^{6,7}$. Durante una infección y como 
parte de la reacción de fase aguda, disminuye la síntesis hepática de albúmina ${ }^{6}$, lo que parece más relacionado con el apremio catabólico pro. vocado por la infección que con el estado nutriciona $^{8}$. Una yez finalizado el estrés catabólico, la concentración plasmática de albúmina aumen. ta, lo que concuerda con nuestros resultados.

La proteina C-reactiva, como signo de daño $t \mathrm{i}$ sular orgánico e índice objetivo de actividad de enfermedad ${ }^{9}$, pareceria tener alta sensibilidad $y$ especificidad para el diagnóstico de infección bacteriana aguda ${ }^{11}$. En nuestro estudio, su comportamiento fue acorde a lo esperado para una reacción de fase aguda, pero no mostró correlación con signos clínicos y radiológicos que sugiriesen infecciones bacterianas o virales. Estas fracciones proteicas son las que contri. buyen, en forma más importante, al aumento de la velocidad de eritrosedimentación ${ }^{10}$ y en esta serie los niveles altos de proteina C-reactiva al ingreso se correlacionaron con alzas de la velocidad de eritrosedimentación, bajando ambos a valores normales al egreso, en los tres grupos. La respuesta a la infeeción en el hombre incluye adaptaciones en el metabolismo de diversos nutrientes, entre ellos la glucosa, para la que se ha descrito intolerancia relativa, con hiperglice. mia $^{12,13}$, que parece causada por aumento de la gluconeogénesis hepática o por resistencia perjférica a la insulina durante la infección o ambas. Los lactantes de los tres grupos presentaron glicemias significativamente más elevada al in. greso, que descendieron a las normales al egreso.

Escogimos la medición de las frecuencias cardiacas y respiratorias como parámetros evaluadores de la falla respiratoria. La medición de] consumo de oxígeno en lactantes es dificil, pero se conoce la relación directa que guarda éste con la frecuencia cardiaca en condiciones normales $o$ de recuperación nutricional ${ }^{14-16}$. La ingesta calórica, aunque no mide el componente de falla respiratoria, aporta información para el estudio de la interacción nutrición-respj. ración. Los músculos respiratorios requieren aporte calórico-proteico adecuado para su función y mantenimiento en condiciones de esfuerzo aumentado ${ }^{17,}{ }^{18}$. Los aportes insuficientes, principalmente energéticos, son uno de los factores contribuyentes al agotamiento de los lactantes con bronconeumonia e insuficiencia respiratoria, to cual puede llevar al empleo de ventilación mecánica. Los grupos alimentados por sonda nasogástrica mostraron significativamente mayor ingestión de calorias que con alimentación ad libitum en los tres primeros días del estudio, sin diferencias a partir del dia 4. Esto se explica por habérseles asegurado, a los lactantes de los grupos A y B, ingresos energéticos vía sonda nasogástrica factibles a pesar de la presencia de falla respiratoria severa. Al mejorar ésta y retirarse la sonda, los tres grupos quedaron alimentándose por boca, igualándose también la ingesta en todos. Sin embargo, algunos efectos, como la más rápida normalización de frecuencia cardíaca en los grupos con alimentación forzada, se hicieron más evidentes a partir del tercer o cuarto dia, después de retirada la sonda, 10 que refuerza el concepto que ellos están mediados por un fenómeno metabólico y no por el ahoro en el esfuerzo de la succión y deglución.

La vía nasogástrica constituye uno de los métodos más prácticos de alimentación gastrointestinal por períodos cortos en el hospital, más aún si hay alteración importante de la función respiratoria a un grado en que comer pudiese acentuar la insuficiencia respiratoria ${ }^{19,20}$, ya que la posibilidad de aspiración es mayor con esta forma de administración; sin embargo, si se toman los cuidados adecuados, como elevar en $30^{\circ}$ la cabecera, es posible disminuir este riesgo en forma importante ${ }^{21,22}$. En nuestro estudio no ocurrieron episodios de aspiración. La mejoría más precoz de los parámetros de función respiratoria en los lactantes de los grupos A y $B$ unidos se tradujo en menor tiempo de hospitalización.

La información conocida sobre apoyo nutricional en enfermedad respiratoria es poca y proviene preferentemente de adultos estables eumetabólicos ${ }^{23}$; sin embargo, existe un activo interés en esta área. Este estudio aporta al conocimiento para el mejor manejo de enfermedades de alta prevalencia en nuestro país con técnicas sencillas. Esto no significa que los hallazgos comentados y los métodos sugeridos sirvan para manejar todas las bronconeumonjas hospitalizadas, pero podría plantearse para las situaciones más graves en los lactantes más pequeños o en aquellos con défjcit nutricional previo.

En síntesis, durante las infecciones respiratorias agudas bajas ocurren alteraciones antropométricas, observándose deterioro en los pacientes dejados en ayuno o semiayuno. Los parámetros bioquímicos estudiados muestran el comportamiento esperado para la reacción de fase aguda de una infección y el apoyo enteral no parece 
influir sobre el comportamiento de ellos. Los niffos alimentados por sonda nasogástrica ingieren más alimentos, tienen menor esfuerzo respiratorio, trabajo metabólico más efectivo, recuperaciones más rápidas $\mathrm{y}$ estadas hospitalarjas más breves. Es necesario continuar con estudios en esta área, con miras a resolver preguntas como si mayores aportes calórico-proteicos que los recibidos por estos lactantes o modificaciones en la proporción de los macronutrientes (proteinas, grasas, hidratos de carbono) inciden en evoluciones aún más favorables de las infecciones respiratorias bajas.

\section{Resumen}

La disminución en la jngesta alimentaria durante la fase aguda de una infección respiratoria baja puede afectar el curso de la enfermedad. Estudiamos 42 lactantes ( 2 a 12 meses) con infección respiratoria baja identificada por los hallazgos clínicos y radiográficos, a los que se les administró antibióticos y nebulizaciones con salbutamol. A su ingreso fueron asignados aleatoriamente al grupa $A$, alimentados por sonda nasogástrica en bolo intemitente; grupo B, alimentados por sonda nasogástrica a goteo continuo, y grupo $\mathrm{C}$, alimentados por boca ad libitum. todos con una fórmula a base de leche de vaca en polvo reconstituida al $7,5 \%$, con sacarosa 5\% y maltodextrina $5 \%(77 \mathrm{kcal} / \mathrm{dl})$. La ingesta energética en el grupo $\mathrm{A}$ aumentó de 69 a $110 \mathrm{kcal} \cdot \mathrm{kg} \cdot$ día; en el grupo B, de 68 a 114 $y$ en el $\mathrm{C}$ de 55 a 109 (p 0,005 para los primeros tres dias). Los lactantes de los grupos A y B tuvieron estadas hospitalarias más corta que los de $\mathrm{C}(8,7$ y 8,4 w 9,8 días; $\mathrm{p}<0,002)$ y recuperación más rápida de los índices de insuficiencia respiratoria $(4,1$ y 3,8 vs 5,5 días; $\mathrm{p}<0,001)$. Los grupos A y B también mostraron mayor descenso de la frecuencia cardiaca media (170 a 134 pulsaciones minuto) que el grupo C $(169$ a $142 ; p<0,01)$. No hubo diferencias en la frecuencia respiratoria, peso/talla, albuminemia, proteína C-reactiva, VHS y glicemia. El apoyo nutricional temprano via sonda nasogástrica parece favorecer la recuperación clínica en lactantes con infecciones agudas del tracto respiratorio bajo.

(Palabras clave: infecciones respiratorias bajas, apoyo nutricional, alimentación enteral, lactan. tes.)

\section{Referencias}

1. Ministerio de Salud: Boletín de egresos hospitalarios por causa y sexo por tramos de edad. Santiago, Chile, 1985.

2. Hospital del Niño: Boletin Estadístico. Panamá, Panamá, 1986.

3. Duffau-Toro, G.; Faúndez-Loyola, J.; Osorio-Sepúlveda, P.: Estado nutricional del lactante hospitalizado por patología respiratoria y digestiva. Estudio prospectivo. Bol Med Hosp Infant Mex 1986; 43: 11-21.

4. Merrit, R.J.; Suskind, R.M.: Nutritional survey of hospitalized pediatric patients. Am J Clin Nutr 1979; 32: 1320-1325.

5. Scrimshaw, H.S.: Significances of the interaction of nutrition and infection in children. En: Textbook of Pediatric Nutrition. Suskind, R.M. (ed), Raven Press, New York 1981; pp. 229-239.

6. Dinarello. C.A.: Intetleukin-1 and the pathogenesis of the acute-phase response. N Engl J Med 1984; 311: 1413-1418.

7. Maiz, A.: Manejo nutricional del paciente crítico. Rev Chil Nutr 1984; 12 : 71-82.

8. Merrit, R.J.; Kalsch, M.+ Roux, L.D.; AshleyMill, J.; Siegel, S.S.: Significance of hypoalbuminemia in pediatric oncology patients-Malnutrition or infection? JPEN 1985; 9: 303-306.

9. Pepys. M.B,; Battz, M.L.: Acute phase proteins with special references to $\mathrm{C}$-reactive protein and related proteins (pentaxins) and serum amyloid A protein. Adv Immunol 1983; 34: 141-211.

10. Tilet. W.S.; Francis Jr., T.: Serologic reactions in pneumonia with a non-protein somatic fractions of pneumococcus. $J$ Exp Med 1930; $52: 561-571$.

11. Stuart, J.; Whicher, J.T.: Tests for detecting and monitoring the acute phase response. Arch Dis Child 1988; 63: 115-117.

12. Beisel, W.R.: Metabolic responses to infection. Ann Rev Med 1975; 26: 9-20.

13. Rayfield, EJ.; Currow, R.T.; George, D.T.; Beisel, W.R.: Impaired carbohydrate metabolism during a mild viral illness. N Engl J Med 1973; 289; 618-621.

14. Spady, D.W.; Payne, P.R.; Picou, D.; Waterlow, $J . C$.: Energy balance during recovery from malnutrition. Am J Clin Nutr 1976; 29: $1073-1088$.

15. Chessex, P.; Reichman, B.; Verellen, G. et al.: Relation between heart rate and energy expenditure in the newborn. Pediatr Res 1981; 15: 1077-1082.

16. Devies, C.T.M.: Limitations to the prediction of maximum oxygen intake from cardiac frequency measurements. J Appl Physiol 1968; 43: 870. 878.

17. Askanazi, J.; Weissman, C.; Rosenboum, S.H.; Hyman, A.I.; Milic-Emili, J.; Kinney, J M.: Nutrition and the respiratory system. Crit Care Med $1982 ; 10: 163-172$.

18. Openbrier, D.R., Irwin, M.M.; Rogers, R.M.; Gottlieb, G.P.; Dauber, J.H.; Van Thiel, D.H.; Pennock, B.E.: Nutritional status and lung function in patients with emphysema and chronic bronchitis. Chest $1983 ; 83: 17-22$. 
19. Bistrian, B.R.; Wade, J.E.: Feeding the hospitalized patient. En: Human Nutrition. Clinical and Biochemical Aspect. Garry, P,J, (ed). The American Association for Clinical Chemistry, Washington, 1981 ; pp. 352-370.

20. Witson, S.E.: Pediatric enteral feeding. En: Pediatric Nutrition. Theory and Practice. Grand, S.J.; Sutphen, J.L.; Dietz, W.H. (eds.). Butterwork Publishers, USA, 1987 ; pp. 771-786.

21. Heymsfield, S.B.; Bethel, R.A.; Ansley, J.D.; Nixon, D.W.; Rudmon, D.: Enteral hyperalimentation. An alternative to control venous hyperalimentation. Ann Intern Med 1979; 90: 63-71.
22. Cotaldi-Betcher, E.L.; Seltzer, M.H.; Slocum, B.A.; Jones, $K . W$.: Complicatjons ocurring during enteral nutrition support: A prospective study. JPEN 1983; 7: 546-552.

23. Heymsfield, S.B.; Casper, K.: Metabolic effects of enteral feeding: Relationship to cardiopulmo. nary complications. En: Enteral feeding: Scientific basis and clinical applications. Balistreri, W.F.; Farrel, M.K. (eds.). Report of the ninety-fourth Ross Conference on Pediatric Research. ColumbusOhio, Ross Laboratories 1988, pp. 35-46. 